Consider those values of $w$ that yield values of $z$ for which $F(z)$ is defined, and for which then $F(z)$ is a function of $w$. These values of $F(z)$ do not constitute an analytic function of $w$; for the domain of values of $w$ consists of two separate continua. Thus the theorem, unrestricted, would be false in this case. *

HARVARD UNIVERSITY, April, 1898.

\title{
NOTE ON POISSON'S INTEGRAL.
}

\author{
BY PROFESSOR MAXIME BÔCHER.
}

(Read before the American Mathematical Society at the Meeting of April $30,1898$.

THe following treatment of Poisson's integral in two dimensions seems to the writer to have at least one advantage over the treatments ordinarily given; viz., that it involves no artifice.

Given a function $V(x, y)$ which within and upon the circumference of a certain circle $C$ is a continuous function of $(x, y)$ and within $C$ is harmonic $(i$. e., has continuous first and second derivatives and satisfies Laplace's equation). By a well-known theorem of Gauss the value of $V$ at the centre $\left(x_{0}, y_{0}\right)$ of $C$ is the arithmetic mean of its values on the circumference. $\dagger$ That is, if we denote by $V_{c}$ the values of $V$ on the circumference and by $\varphi$ the angle at the centre,

$$
V\left(x_{0}, y_{0}\right)=\frac{1}{2 \pi} \int_{0}^{2 \pi} V_{c} d \varphi \text {. }
$$

This theorem may be immediately generalized by the method of inversion, if we remember on the one hand that a harmonic function remains harmonic after inversion, and on the other hand that angles are unchanged by inversion and that circles invert into circles. We thus get the theorem :

* Burkhardt has given simple examples of multiple-valued functions for which the unrestricted theorem is false. See his book : "Einführung in die Theorie der analytischen Functionen einer complexen Veränderlichen," vol. 1, Leipzig, 1897 ; p. 198.

$\dagger$ An elementary proof of this theorem will be found in a paper by the writer on p. 206 of the BuLLETIN for May, 1895. 
If $(x, y)$ is any point within the circle $C$,

$$
V(x, y)=\frac{1}{2 \pi} \int_{0}^{2 \pi} V_{c} d \psi
$$

where $\psi$ is the angle measured from a fixed circle through $(x, y)$ which cuts $C$ orthogonally to a variable circle of the same sort.

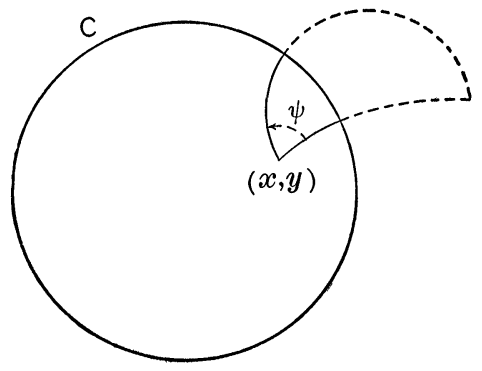

The formula (2) gives the value of $V$ at any point within $C$ in terms of the values on the circumference, and is, in fact, nothing but Poisson's integral in a somewhat unfamiliar form. To reduce it to the ordinary forms we will introduce the element of arc $d s$ of $C$ and write (2) in the form :

$$
V(x, y)=\frac{1}{2 \pi} \int V_{c} \frac{\partial \psi}{\partial s} d s .
$$

$\psi$ may be regarded as a function of the coördinates $\left(x^{\prime}, y^{\prime}\right)$ of a point on the variable circle above referred to. Thus regarded it is an (infinitely multiple valued) solution of Taplace's equation whose conjugate function is $\log \rho_{1}-\log \rho$ where $\rho$ is the distance from $\left(x^{\prime}, y^{\prime}\right)$ to $(x, y)$ and $\rho_{1}$ the distance from $\left(x^{\prime}, y^{\prime}\right)$ to the inverse of $(x, y)$ with regard to $C$. If then $n$ denote the interior normal of $C$ we have :

$$
V(x, y)=\frac{1}{2 \pi} \int V_{c} \frac{\partial\left[\log \rho_{1}-\log \rho\right]}{\partial n} d s . *
$$

Other forms can be obtained by computing $\frac{\partial \psi}{\partial s}$ directly as a function of $\left(x, y, x^{\prime}, y^{\prime}\right)$.

We proceed now to the theorem :

Given a continuous function $V_{c}$ upon the circumference of the circle $C$; the function $V(x, y)$ defined by (2) throughout the interior of $C$ is harmonic throughout $C$ and joins on continuously to the values $V_{c}$ on the circumference.

\footnotetext{
* Cf. Picard : Traité d'Analyse, vol. 2, p. 16.
} 
That $V(x, y)$ thus defined is harmonic follows at once from (3) since $\frac{\partial \psi}{\partial s}$ is easily seen, either by direct computation or from its value :

$$
\frac{\partial}{\partial n}\left[\log \rho_{1}-\log \rho\right]
$$

to be a harmonic function of $(x, y)$.

For the proof of the second part of the theorem formula (2) is particularly adapted. We have here to prove that if $(x, y)$ approaches a point $P$ on the circumference $V(x, y)$ approaches as its limit the value of $V_{c}$ at $P$. The idea upon which this proof rests is that when $(x, y)$ is near to $P$ a small arc including $P$ corresponds to a large range of values of $\psi$ and, therefore, when we take the arithmetic mean as indicated in (2) the value of $V_{\text {c }}$ at $P$ will predominate.* The exact proof based upon the idea just stated merely requires the writing down of a few inequalities.

HaRvard University, CaMbridge, Mass.

\section{ON THE POLYNOMIALS OF STIELTJES.}

BY PROFESSOR K. B. VAN VLECK.

(Read before the American Mathematical Society at the Meeting of April 30, 1898.)

By a Stieltjes polynomial will here be understood any polynomial satisfying a regular linear differential equation of the second order

$$
\begin{gathered}
\frac{d^{2} y}{d x^{2}}+\left(\frac{1-\lambda_{1}}{x-e_{1}}+\cdots+\frac{1-\lambda_{r}}{x-e_{r}}\right) \frac{d y}{d x} \\
+\frac{\varphi(x)=A_{0} x^{r-2}+A_{1} x^{r-3}+\cdots+A_{r-2}}{\left(x-e_{1}\right) \cdots\left(x-e_{r}\right)} y=0
\end{gathered}
$$

in which the singular points $e_{1}, \cdots, e_{r}, \infty$ are real and in which also $r$ exponent-differences $\lambda_{1}, \cdots,,_{r}$ are (algebraically) less than unity. We shall here for the most part confine our

* It will be seen that this idea is similar to that suggested by Schwarz. (Ges. Werke, vol. 2, p. 360. See also Klein-Fricke: Modulfunctionen, vol. 1, p. 512.) We avoid, however, the artificiality of Schwarz's method. 